Annuaire suisse de politique de développement

23-1 | 2004

Faits et statistiques 2004

\title{
1. Commerce
}

\section{Christoph Stamm et Gérard Perroulaz}

\section{OpenEdition \\ Journals}

Édition électronique

URL : http://journals.openedition.org/aspd/487

DOI : 10.4000/aspd.487

ISSN : 1663-9669

\section{Éditeur}

Institut de hautes études internationales et du développement

\section{Édition imprimée}

Date de publication : 1 avril 2004

Pagination : 241-259

ISSN : 1660-5934

\section{Référence électronique}

Christoph Stamm et Gérard Perroulaz, «1. Commerce », Annuaire suisse de politique de développement [En ligne], 23-1 | 2004, mis en ligne le 16 mars 2010, consulté le 08 septembre 2020. URL : http:// journals.openedition.org/aspd/487 ; DOI : https://doi.org/10.4000/aspd.487 


\section{Commerce}

\section{Commentaire}

\subsection{Structure géographique du commerce extérieur de la Suisse}

(Tableaux de la partie 1.1)

En raison de la faible conjoncture nationale et internationale, la valeur des échanges commerciaux de la Suisse a diminué en 2002 par rapport à 2001. Après deux ans de solde commercial déficitaire, c'est la forte baisse des importations qui a permis à la Suisse de réaliser un excédent de la balance commerciale très important d'un montant de 6,3 milliards de francs en 2002.

Les principaux partenaires commerciaux de la Suisse sont les pays de l'Union européenne et les Etats-Unis. En 2002, $88 \%$ des importations de la Suisse provenaient de pays développés, et ceux-ci représentaient des débouchés pour $78 \%$ des exportations suisses (tableau 1.1.A.2). 58\% des importations de la Suisse proviennent de l'Allemagne, de la France, de l'Italie et des Etats-Unis et $49 \%$ des exportations sont destinées à ces pays (tableau 1.1.C.1).

La Suisse réalise un important excédent commercial dans ses échanges avec les pays en développement (le tiers-monde) (+ 13,8 milliards de francs en 2002) alors que le déficit de la balance commerciale avec les pays européens industrialisés s'élève à 20,8 milliards de francs (tableau 1.1.A.2). L'excédent commercial est particulièrement important avec les pays asiatiques (11,4 milliards de francs en 2002) (tableau 1.1.B.2).

La part de l'Asie dans le commerce de la Suisse avec le tiers-monde est prépondérante. Comme le montrent les graphiques 2, $65 \%$ des importations de la Suisse en provenance des pays du tiers-monde viennent du continent asiatique, auquel $74 \%$ des exportations sont destinées. 


\section{Graphiques 2: Commerces de la Suisse avec les pays du tiers-monde, année 2002}

Répartition par continent (en \% du total des importations et des exportations de et vers le tiers-monde)

Importations (11'791,5 mio. fr.)

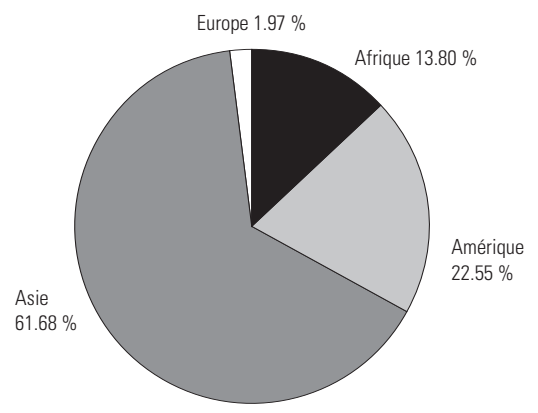

Exportations (25'611,7 mio. fr.)

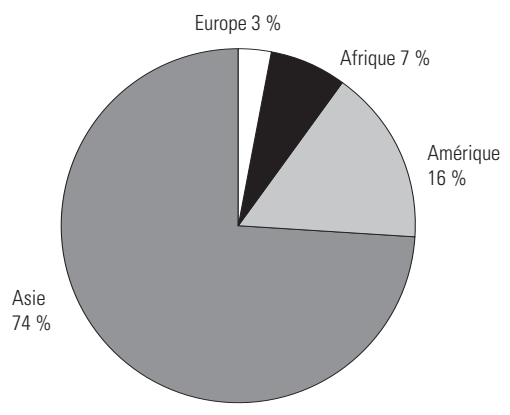

Sources: Direction générale des douanes, Commerce extérieur de la Suisse 2002, vol. 4/4, 2002, Statistiques selon les marchandises et les pays, Berne, 2003, pp. 854-859, <www.zoll.admin.ch>.

Deux remarques s'imposent:

$\square$ Nous appliquons dans cette partie statistique la classification des pays établie par la CNUCED. La situation se présente un peu différemment lorsque l'on tient compte, comme dans la partie 4 de ces statistiques, des classifications des pays établies par le CAD (pays en développement et pays en transition, cette catégorie comprenant les «pays en développement plus avancés»). La part de l'Asie est ici plus importante que dans la partie 4 des statistiques ou dans les graphiques des pages précédentes (introduction). Cela s'explique essentiellement par le fait que dans les graphiques d'introduction et les tableaux de la partie 4, plusieurs pays asiatiques (par exemple la Corée du Sud, Hongkong, Macao, Singapour et Taiwan) sont classés par le CAD dans le groupe des pays en développement plus avancés, alors que dans les graphiques 1 et 2 ils apparaissent sous «Asie».

$\square$ Le montant des importations de matières premières et de produits énergétiques en provenance du tiers-monde est faible. Une grande partie des matières premières originaires du tiers-monde ont subi des transformations dans des pays industrialisés avant de parvenir en Suisse. Ces produits sont enregistrés comme des importations en provenance de ces derniers pays (en particulier des pays voisins de la Suisse). Les exportations suisses se composent essentiellement de produits transformés, souvent très élaborés et à haute valeur ajoutée. Ce type de production correspond à la spécialisation de l'industrie suisse. Compte tenu également du bas prix de certaines importations, il n'est pas étonnant que le solde des échanges avec le tiers-monde de produits intermédiaires et de matières premières soit positif, (voir commerce des matières premières et demi-produits, tableau 1.3.A.1).

La part de l'Afrique dans le commerce extérieur de la Suisse est très faible. Le commerce avec tous les pays africains (y compris l'Afrique du Nord) représente 1,2\% pour les importations et $1,3 \%$ pour les exportations du total des échanges extérieurs de la Suisse. 
Seulement 0,11\% des importations de la Suisse proviennent des PMA, qui représentent un débouché pour $0,39 \%$ des exportations suisses (tableau 1.1.B.2). La part des PMA dans les importations de la Suisse est stagnante ces dernières années, alors que les exportations de la Suisse vers ces pays ont fortement augmenté, passant de 267 à 530,1 millions de francs de 1999 à 2002.

Le tableau 1.1.C.2 montre les 15 principaux partenaires commerciaux dans le tiersmonde. La Chine est de loin le principal fournisseur parmi les pays du Sud (18,7\% des importations). Si on compare les principaux fournisseurs en 2002 avec ceux en 1992, il apparaît que 4 pays, les Bermudes, le Panamá, l'Argentine et l'Indonésie, ne font plus partie des 15 plus grands fournisseurs et ont été remplacés par la Chine, Porto Rico, le Nigeria et la Colombie.

Le client de loin le plus important est Hongkong (17,5\% des exportations). Si on compare les principaux clients en 2002 avec ceux d'il y a dix ans, ce sont deux pays qui'ont changé. L'Indonésie et le Pakistan ont été remplacés par la Chine et l'Egypte.

\subsection{Evolution du commerce avec certains pays partenaires dans le tiers- monde, 1993-2002}

Les graphiques de la partie 1.1.D montrent l'évolution du commerce avec certains pays partenaires importants. Pour la plupart des partenaires commerciaux de la Suisse (la Turquie et le Mexique par exemple), les exportations de la Suisse vers ces pays dépassent largement les importations qui en proviennent - à l'exception de la Chine. Depuis quelques années, la Suisse a envers ce pays un déficit commercial important, mais depuis trois ans, les exportations vers la Chine augmentent beaucoup plus rapidement que les importations provenant de ce pays, ce qui laisse prévoir un changement de tendance.

Dans le cas de la Thaïlande, on peut observer l'impact de la crise asiatique de 1997 qui avait fortement touché ce pays. La Suisse exporte par la suite (1998) beaucoup moins de marchandises vers la Thaillande alors que les importations restent très stables. Même en 2002, la Suisse exporte toujours moins qu'elle ne le faisait au milieu des années 1990.

L'Afrique du Sud constitue un autre cas intéressant: l'important excédent commercial qu'a la Suisse avec ce pays au début des années 1990 se réduit pour devenir, à partir de 2001, un déficit commercial.

Le déficit commercial important qu'a la Suisse avec la Russie (ce pays n'est pas classé comme pays du tiers-monde) depuis quelques années s'explique avant tout par les importations très élevées dans la catégorie des métaux précieux/pierres gemmes.

\subsection{Composition des échanges de la Suisse avec le tiers-monde}

(Tableaux de la partie 1.2)

Les importations suisses en provenance du tiers-monde comprennent essentiellement des biens de consommation ( $45 \%$ des importations), des matières premières et demi-produits $(22 \%)$ et des biens d'équipement (14\%). La Suisse exporte vers ces pays surtout des biens de consommation ( $42 \%$ des exportations), des biens d'équipement $(32 \%)$ et des demi-produits $(19 \%)$. 


\subsection{Structure géographique du commerce extérieur de la Suisse}

\section{A. Principaux courants commerciaux : importations, exportations, solde de la balance commerciale}

\section{Année 2001}

\begin{tabular}{lrrrrrr}
\hline \multirow{2}{*}{ Groupes de pays } & \multicolumn{2}{c}{ Importations } & & Exportations & Solde & $\begin{array}{r}\text { Coeff. } \\
\text { échange }\end{array}$ \\
\cline { 2 - 8 } & Mio Fr. & $\%$ & Mio Fr. & $\%$ & Mio Fr. & Exp./Imp. \\
\hline Monde & $\mathbf{1 4 1 8 8 8 , 9}$ & $\mathbf{1 0 0 , 0}$ & $\mathbf{1 3 8 4 9 1 , 1}$ & $\mathbf{9 9 , 5}$ & $\mathbf{- 3 3 9 7 , 8}$ & $\mathbf{0 , 9 8}$ \\
\hline $\begin{array}{l}\text { Pays développés } \\
\text { à économie de marché }\end{array}$ & 122194,4 & 86,1 & 109221,9 & 78,9 & $-12972,5$ & 0,89 \\
\hline \multicolumn{1}{c}{ Europe } & 108559,4 & 76,5 & 83777,5 & 59,0 & $-24781,9$ & 0,77 \\
\hline \multicolumn{1}{c}{ Outre-mer } & 13635,0 & 9,6 & 25444,4 & 17,9 & 11809,4 & 1,87 \\
\hline Pays d'Europe orientale & 7047,5 & 5,0 & 4768,5 & 3,4 & $-2279,0$ & 0,68 \\
\hline Pays du tiers-monde & 12647,0 & 8,9 & 24500,7 & 17,3 & 11853,7 & 1,94 \\
\hline
\end{tabular}

\section{Année 2002}

\begin{tabular}{lrrrrrr}
\hline \multirow{2}{*}{ Groupes de pays } & \multicolumn{2}{c}{ Importations } & \multicolumn{2}{c}{ Exportations } & Solde & $\begin{array}{r}\text { Coeff. } \\
\text { échange }\end{array}$ \\
\cline { 2 - 8 } & Mio Fr. & $\%$ & Mio Fr. & $\%$ & Mio Fr. & Exp./Imp. \\
\hline Monde & $\mathbf{1 3 0 1 9 3 , 2}$ & $\mathbf{1 0 0 , 0}$ & $\mathbf{1 3 6 5 2 2 , 9}$ & $\mathbf{1 0 0 , 0}$ & $\mathbf{6 3 2 9 , 7}$ & $\mathbf{1 , 0 5}$ \\
\hline $\begin{array}{l}\text { Pays développés } \\
\text { à économie de marché }\end{array}$ & 114353,4 & 87,8 & 106126,4 & 77,7 & $-8227,0$ & 0,93 \\
\hline \multicolumn{1}{c}{ Europe } & 101966,5 & 78,3 & 81186,6 & 59,5 & $-20779,9$ & 0,80 \\
\hline \multicolumn{1}{c}{ Outre-mer } & 12386,9 & 9,5 & 24939,8 & 18,3 & 12552,9 & 2,01 \\
\hline Pays d'Europe orientale & 4048,4 & 3,1 & 4784,9 & 3,5 & 736,5 & 1,18 \\
\hline Pays du tiers-monde & 11791,4 & 9,1 & 25611,6 & 18,8 & 13820,2 & 2,17 \\
\hline
\end{tabular}

Source tableaux 1.1.A et 1.1.B: Direction générale des douanes, Commerce extérieur de la Suisse 2002, vol. 4/4, 2002, Statistiques selon les marchandises et les pays, Berne, 2003, pp. 854-859. 


\subsection{Structure géographique du commerce extérieur de la Suisse (suite)}

B. Commerce avec le tiers-monde : importations, exportations, solde de la balance commerciale

\section{Année 2001}

\begin{tabular}{|c|c|c|c|c|c|c|}
\hline \multirow{2}{*}{ Groupes de pays ${ }^{1}$} & \multicolumn{2}{|c|}{ Importations } & \multicolumn{2}{|c|}{ Exportations } & \multirow{2}{*}{$\begin{array}{r}\text { Solde } \\
\text { Mio Fr. }\end{array}$} & \multirow{2}{*}{$\begin{array}{r}\begin{array}{r}\text { Coeff. } \\
\text { échang }\end{array} \\
\text { Exp./Imp. }\end{array}$} \\
\hline & Mio Fr. & $\%^{2}$ & Mio Fr. & $\%^{2}$ & & \\
\hline Ensemble pays tiers-monde & 12647,0 & 8,9 & 24500,7 & 17,3 & 11853,7 & 1,94 \\
\hline \multicolumn{7}{|l|}{ a) Groupes géographiques } \\
\hline Afrique & 1745,3 & 1,2 & 1630,3 & 1,2 & $-115,0$ & 0,93 \\
\hline Amérique & 2850,5 & 2,0 & 4672,4 & 3,4 & 1821,9 & 1,64 \\
\hline Asie & 7798,3 & 5,5 & 17483,3 & 12,6 & 9685,0 & 2,24 \\
\hline Europe & 248,7 & 0,2 & 687,7 & 0,5 & 439,0 & 2,77 \\
\hline \multicolumn{7}{|l|}{ b) Groupes économiques } \\
\hline Export. de pétrole & 2104,7 & 1,5 & 3971,7 & 2,9 & 1867,0 & 1,89 \\
\hline Export. d'art. manuf. & 7537,7 & 5,3 & 15457,3 & 11,2 & 7919,7 & 2,05 \\
\hline Autres pays & 3004,6 & 2,1 & 5071,8 & 3,7 & 2067,2 & 1,69 \\
\hline c) Pays les moins avancés & 147,4 & 0,1 & 378,3 & 0,3 & 230,9 & 2,57 \\
\hline
\end{tabular}

\section{Année 2002}

\begin{tabular}{lrrrrrr}
\hline & \multicolumn{2}{c}{ Importations } & Exportations & Solde & $\begin{array}{r}\text { Coeff. } \\
\text { échange }\end{array}$ \\
\cline { 2 - 7 } Groupes de pays ${ }^{1}$ & Mio Fr. & $\boldsymbol{\%}^{2}$ & Mio Fr. & $\boldsymbol{\%}^{2}$ & Mio Fr. & Exp./Imp. \\
& & & & & & \\
\hline Ensemble pays tiers-monde & $\mathbf{1 1 7 9 1 , 4}$ & $\mathbf{9 , 1}$ & $\mathbf{2 5 6 1 1 , 6}$ & $\mathbf{1 8 , 8}$ & $\mathbf{1 3 8 2 0 , 2}$ & $\mathbf{2 , 1 7}$ \\
\hline a) Groupes géographiques & & & & & & \\
\hline Afrique & 1584,3 & 1,2 & 1729,8 & 1,3 & 145,5 & 1,09 \\
\hline Amérique & 2412,3 & 1,9 & 4177,6 & 3,1 & 1765,3 & 1,73 \\
\hline Asie & 7528,9 & 5,8 & 18928,8 & 13,9 & 11399,9 & 2,51 \\
\hline Europe & 264,0 & 0,2 & 748,1 & 0,5 & 484,1 & 2,83 \\
\hline b) Groupes économiques & & & & & & \\
\hline Export. de pétrole & 2005,3 & 1,5 & 4518,7 & 3,3 & 2513,4 & 2,25 \\
\hline Export. d'art. manuf. & 7155,3 & 5,5 & 16132,8 & 11,8 & 8977,5 & 2,25 \\
\hline \multicolumn{1}{c}{ Autres pays } & 2630,8 & 2,0 & 4960,1 & 3,6 & 2329,3 & 1,89 \\
\hline c) Pays les moins avancés & 149,6 & 0,1 & 530,1 & 0,4 & 380,5 & 3,54 \\
\hline
\end{tabular}

${ }^{1}$ La composition des groupes de pays figure dans l'Appendice au point 5.2.

${ }^{2}$ Part du commerce extérieur de la Suisse (voir total des échanges avec le monde, tableaux 1.1.A). 


\subsection{Structure géographique du commerce extérieur de la Suisse (suite) \\ C. Principaux fournisseurs et clients de la Suisse \\ 1. Les 15 premiers partenaires commerciaux dans le monde (2002)}

\begin{tabular}{|c|c|c|c|c|c|c|c|}
\hline \multicolumn{4}{|c|}{ Principaux fournisseurs } & \multicolumn{4}{|c|}{ Principaux clients } \\
\hline \multicolumn{4}{|c|}{ Importations } & \multicolumn{4}{|c|}{ Exportations } \\
\hline Pays $^{1}$ & Mio Fr. & $\%$ & $\sum \%$ & Pays $^{1}$ & Mio Fr. & $\%$ & $\sum \%$ \\
\hline 1. Allemagne & 40868,7 & 31,39 & 31,39 & 1. Allemagne & 27876,3 & 20,42 & 20,42 \\
\hline 2. Italie & 13414,8 & 10,30 & 41,69 & 2.USA & 15880,0 & 11,63 & 32,05 \\
\hline 3. France & 13014,9 & 10,00 & 51,69 & 3. France & 12450,1 & 9,12 & 41,17 \\
\hline 4. USA & 7987,1 & 6,13 & 57,83 & 4. Italie & 11215,6 & 8,22 & 49,39 \\
\hline 5.Pays-Bas & 6651,6 & 5,11 & 62,93 & 5. Gr. Bretagne & 7011,2 & 5,14 & 54,52 \\
\hline 6. Gr. Bretagne & 6315,1 & 4,85 & 67,78 & 6. Japon & 5179,4 & 3,79 & 58,32 \\
\hline 7. Autriche & 5273,7 & 4,05 & 71,84 & 7. Autriche & 4543,1 & 3,33 & 61,64 \\
\hline 8. Irlande & 5131,7 & 3,94 & 75,78 & 8. Espagne & 4528,1 & 3,32 & 64,96 \\
\hline 9. Belgique & 3852,5 & 2,96 & 78,74 & 9. Hongkong & 4479,7 & 3,28 & 68,24 \\
\hline 10. Espagne & 2635,3 & 2,02 & 80,76 & 10. Pays-Bas & 4092,2 & 3,00 & 71,24 \\
\hline 11.Japon & 2619,1 & 2,01 & 82,77 & 11. Belgique & 2851,2 & 2,09 & 73,33 \\
\hline 12. Chine & 2206,9 & 1,70 & 84,47 & 12. Chine & 2046,5 & 1,50 & 74,83 \\
\hline 13. Russie & 1527,1 & 1,17 & 85,64 & 13. Turquie & 1586,8 & 1,16 & 75,99 \\
\hline 14. Suède & 1494,6 & 1,15 & 86,79 & 14. Canada & 1547,1 & 1,13 & 77,12 \\
\hline 15. Danemark & 1107,6 & 0,85 & 87,64 & 15. Singapour & 1518,1 & 1,11 & 78,23 \\
\hline Autres pays & 16092,6 & 12,36 & 100,00 & Autres pays & 29717,7 & 21,77 & 100,00 \\
\hline Total & 130193,3 & & 100,00 & Total & 136522,9 & & 100,00 \\
\hline
\end{tabular}

Sources pour les tableaux 1.1.C.1 à 1.3.F.2: Direction générale des douanes, Commerce extérieur de la Suisse 2002, vol. 4/4, 2002, Statistiques selon les marchandises et les pays, Berne, 2003, pp. 854-859.

\footnotetext{
${ }^{1}$ Les pays du tiers-monde sont en caractères gras.
} 


\subsection{Structure géographique du commerce extérieur de la Suisse (fin)}

C. Principaux fournisseurs et clients de la Suisse

2. Les 15 premiers partenaires commerciaux dans le tiers-monde (2002) ${ }^{1}$

\begin{tabular}{|c|c|c|c|c|c|c|c|}
\hline \multicolumn{4}{|c|}{ Principaux fournisseurs } & \multicolumn{4}{|c|}{ Principaux clients } \\
\hline \multicolumn{4}{|c|}{ Importations } & \multicolumn{4}{|c|}{ Exportations } \\
\hline Pays & Mio Fr. & $\%$ & $\sum \%$ & Pays & Mio Fr. & $\%$ & $\sum \%$ \\
\hline 1. Chine & 2206,9 & 18,72 & 18,72 & 1. Hongkong & 4479,7 & 17,49 & 17,49 \\
\hline 2. Hongkong & 824,4 & 6,99 & 25,71 & 2.Chine & 2046,5 & 7,99 & 25,48 \\
\hline 3. Brésil & 706,5 & 5,99 & 31,70 & 3. Turquie & 1586,8 & 6,20 & 31,68 \\
\hline 4. Libye & 657,8 & 5,58 & 37,28 & 4. Singapour & 1518,1 & 5,93 & 37,60 \\
\hline 5. Porto Rico & 618,3 & 5,24 & 42,52 & 5. Taiwan & 1185,2 & 4,63 & 42,23 \\
\hline 6. Taiwan & 604,7 & 5,13 & 47,65 & 6. Arabie Saoudite & 1176,0 & 4,59 & 46,82 \\
\hline 7. Thailande & 601,5 & 5,10 & 52,75 & 7. Brésil & 1103,9 & 4,31 & 51,13 \\
\hline 8. Corée du Sud & 534,6 & 4,53 & 57,29 & 8. Corée du Sud & 1096,4 & 4,28 & 55,41 \\
\hline 9. Inde & 516,1 & 4,38 & 61,66 & 9. Mexique & 990,1 & 3,87 & 59,28 \\
\hline 10. Nigéria & 426,3 & 3,62 & 65,28 & 10. Emirats Arabes & 945,3 & 3,69 & 62,97 \\
\hline 11. Turquie & 413,4 & 3,51 & 68,79 & 11. Thailande & 802,8 & 3,13 & 66,11 \\
\hline 12. Colombie & 296,5 & 2,51 & 71,30 & 12. Inde & 641,3 & 2,50 & 68,61 \\
\hline 13. Singapour & 250,0 & 2,12 & 73,42 & 13. Iran & 481,3 & 1,88 & 70,49 \\
\hline 14. Arabie Saoudite & 229,4 & 1,95 & 75,37 & 14. Egypte & 470,7 & 1,84 & 72,33 \\
\hline 15. Malaisie & 198,4 & 1,68 & 77,05 & 15. Malaisie & 445,6 & 1,74 & 74,07 \\
\hline Reste du tiers-monde & 2706,7 & 22,96 & 100,00 & Reste du tiers-monde & 6641,9 & 25,93 & 100,00 \\
\hline Total & 11791,4 & & 100,00 & Total & 25611,6 & & 100,00 \\
\hline
\end{tabular}

1 Voir aussi les tableaux 4.1 et 4.2 qui contiennent les chiffres du commerce de la Suisse avec tous les pays en développement et en transition selon la classification CAD (Comité d'aide au développement de l'OCDE). 


\subsection{Stucture géographique du commerce extérieur de la Suisse}

D. Evolution du commerce avec quelques pays partenaires commerciaux, en millions de francs (1993-2002)

\section{Ensemble des pays du tiers-monde}

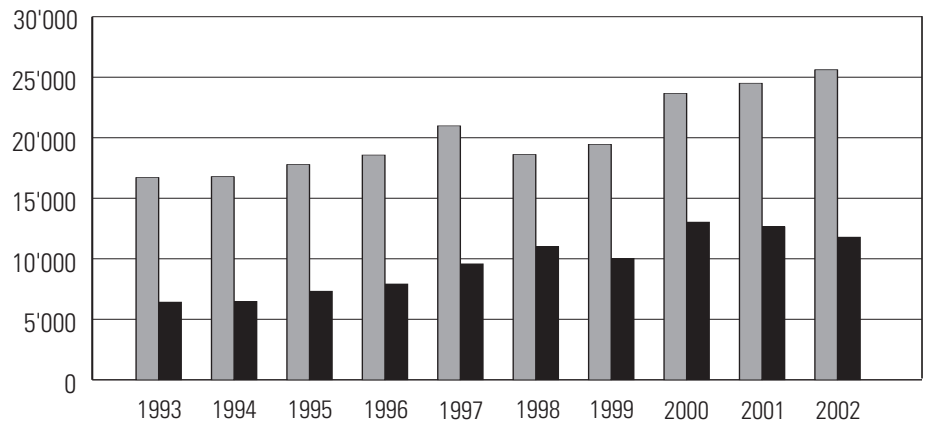

Fédération de Russie

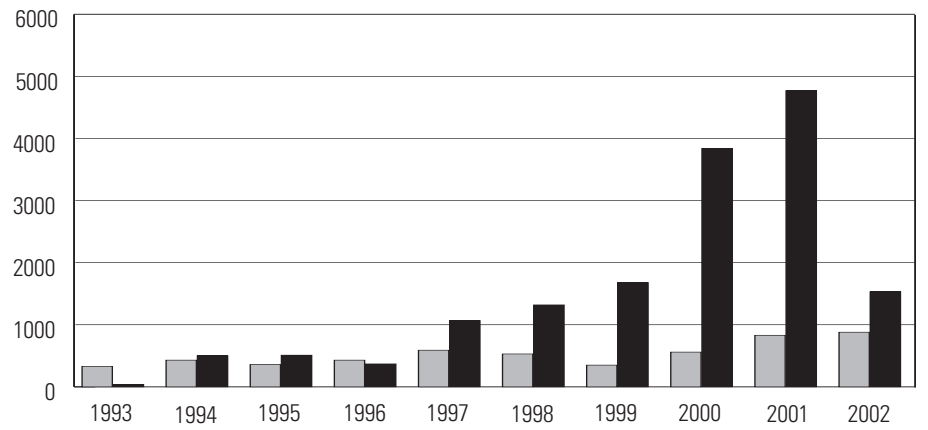

Chine

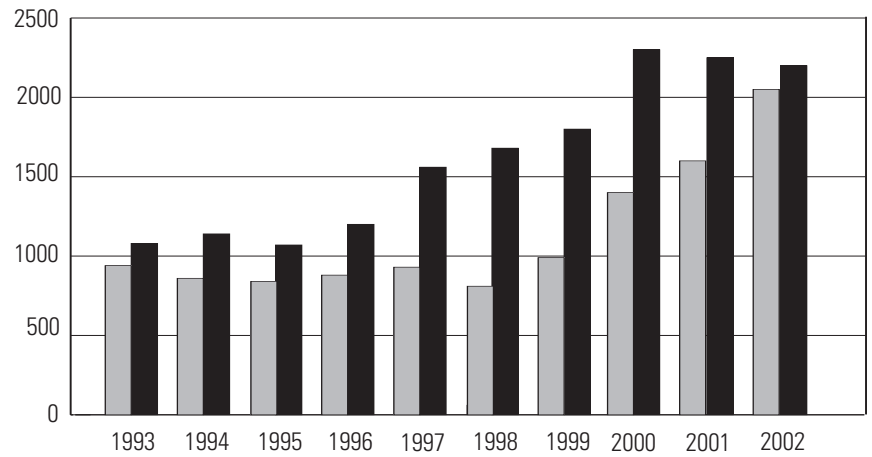

Exportations de la Suisse vers le pays partenaire.

Importations de la Suisse en provenance du pays partenaire.

La différence entre les deux représente l'excédent commercial de la Suisse (lorsque les exportations dépassent les importations en provenance du pays partenaire) ou du déficit commercial (lorsque les importations dépassent les exportations). 
1.1. Stucture géographique du commerce extérieur de la Suisse (suite)

D. Evolution du commerce avec les pays partenaires commerciaux dans le tiers-monde, en millions de francs

\section{Turquie}

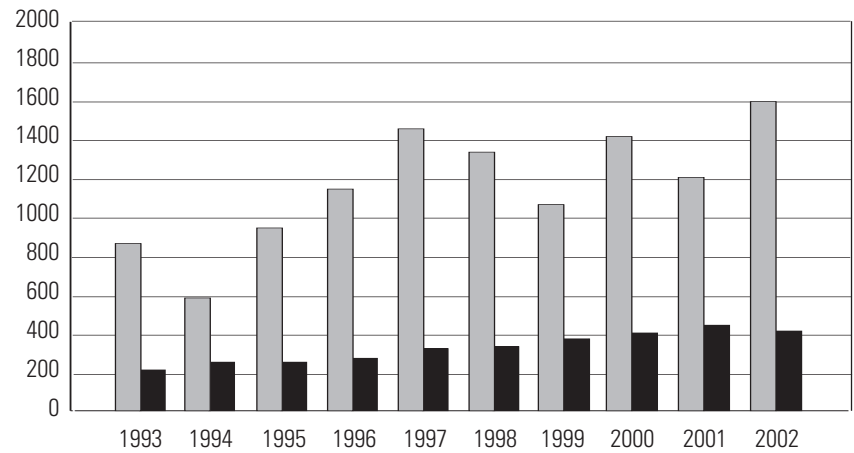

\section{Brésil}

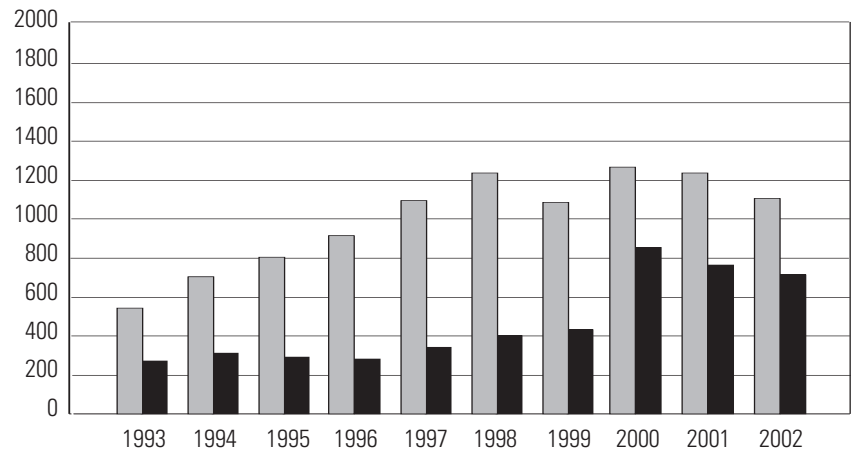

\section{Afrique du Sud}

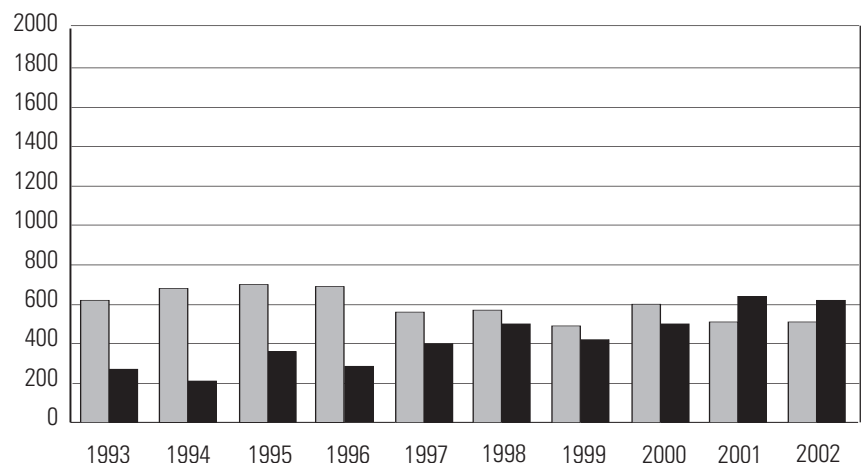

Exportations de la Suisse vers le pays partenaire.

Importations de la Suisse en provenance du pays partenaire. 
1.1. Stucture géographique du commerce extérieur de la Suisse (fin)

D. Evolution du commerce avec les pays partenaires commerciaux dans le tiers-monde, en millions de francs

\section{Thaillande}

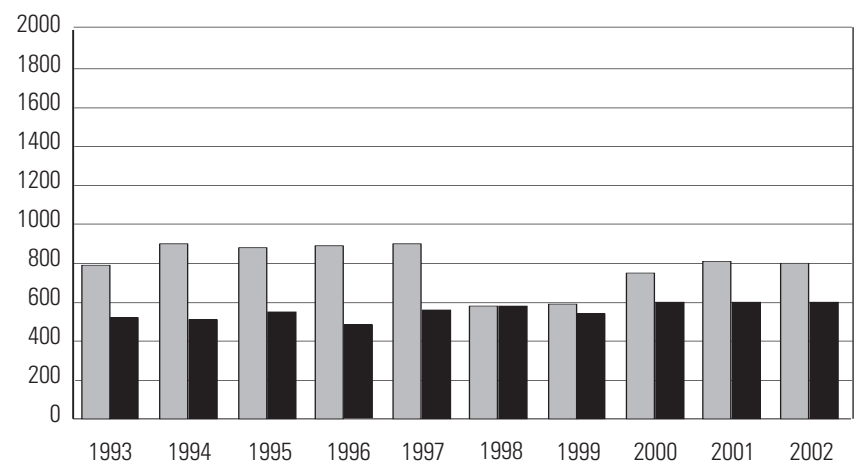

Inde

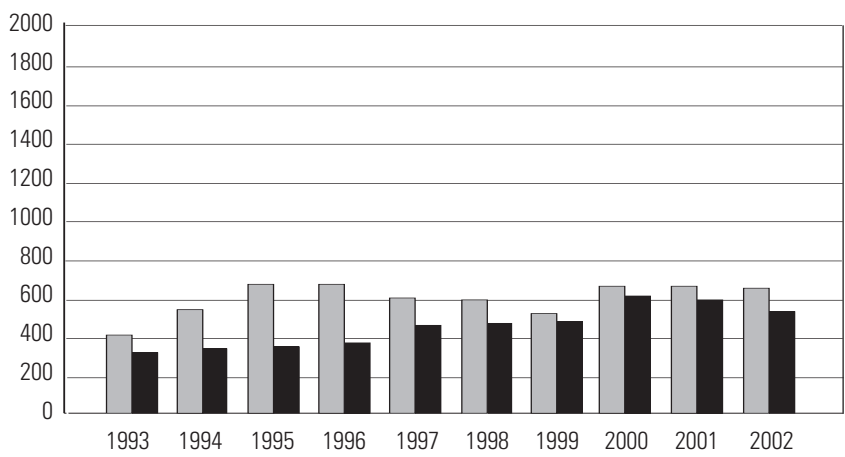

\section{Mexique}

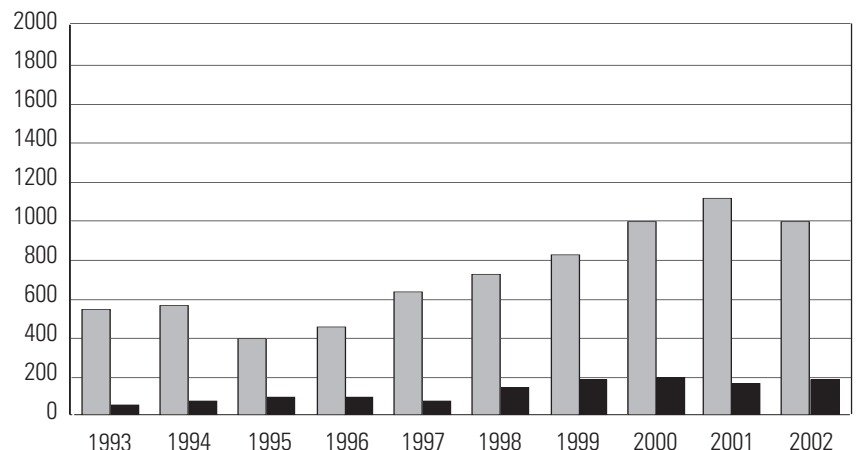

Exportations de la Suisse vers le pays partenaire.

Importations de la Suisse en provenance du pays partenaire. 


\subsection{Composition des échanges de la Suisse avec le tiers-monde}

A. Ensemble des pays du tiers-monde (2002)

\begin{tabular}{lrrrrr}
\hline \multirow{2}{*}{$\begin{array}{l}\text { Catégorie de } \\
\text { marchandises }\end{array}$} & \multicolumn{2}{c}{ Importations } & \multicolumn{2}{c}{ Exportations } & Solde \\
\cline { 2 - 6 } & Mio Fr. & $\%$ & Mio Fr. & \% & Mio $\mathbf{~ r r}$ \\
\hline Matières premières + demi-produits & 2601,2 & 22,1 & 4803,2 & 18,8 & 2202,0 \\
\hline Produits énergétiques & 1420,7 & 12,0 & 77,3 & 0,3 & $-1343,4$ \\
\hline Biens d'équipement & 1651,6 & 14,0 & 8156,0 & 31,8 & 6504,4 \\
\hline Biens de consommation & 5261,7 & 44,6 & 10751,7 & 42,0 & 5490,0 \\
\hline Métaux précieux/pierres gemmes & 742,3 & 6,3 & 1737,8 & 6,8 & 995,5 \\
\hline Objets d'art/antiquités & 114,0 & 1,0 & 85,5 & 0,3 & $-28,5$ \\
\hline Total & $\mathbf{1 1 7 9 1 , 5}$ & $\mathbf{1 0 0 , 0}$ & $\mathbf{2 5 6 1 1 , 5}$ & $\mathbf{1 0 0 , 0}$ & $\mathbf{1 3 8 2 0 , 0}$ \\
\hline
\end{tabular}

\section{B. Ensemble du monde (2002)}

\begin{tabular}{lrrrrr}
\hline \multirow{2}{*}{$\begin{array}{l}\text { Catégorie de } \\
\text { marchandises }\end{array}$} & \multicolumn{2}{c}{ Importations } & Exportations & Solde \\
\cline { 2 - 6 } & Mio Fr. & $\%$ & Mio Fr. & $\%$ & Mio Fr. \\
\hline Matières premières + demi-produits & 32241,7 & 24,8 & 32742,0 & 24,0 & 500,3 \\
\hline Produits énergétiques & 5368,7 & 4,1 & 362,9 & 0,3 & $-5005,8$ \\
\hline Biens d'équipement & 32706,0 & 25,1 & 42256,6 & 31,0 & 9550,6 \\
\hline Biens de consommation & 52808,6 & 40,6 & 55019,0 & 40,3 & 2210,4 \\
\hline Métaux précieux/pierres gemmes & 5961,8 & 4,6 & 4805,9 & 3,5 & $-1155,9$ \\
\hline Objets d'art/antiquités & 1106,5 & 0,8 & 1336,6 & 1,0 & 230,1 \\
\hline Total & $\mathbf{1 3 0 1 9 3 , 3}$ & $\mathbf{1 0 0 , 0}$ & $\mathbf{1 3 6 5 2 3 , 0}$ & $\mathbf{1 0 0 , 0}$ & $\mathbf{6 3 2 9 , 7}$ \\
\hline
\end{tabular}

\section{Principaux exportateurs de pétrole (2002)}

\begin{tabular}{lrrrrr}
\hline \multirow{2}{*}{$\begin{array}{l}\text { Catégorie de } \\
\text { marchandises }\end{array}$} & \multicolumn{2}{c}{ Importations } & \multicolumn{2}{c}{ Exportations } & Solde \\
\cline { 2 - 7 } & Mio Fr. & $\%$ & Mio Fr. & $\%$ & Mio $\mathbf{~ r r}$ \\
\hline Matières premières + demi-produits & 79,2 & 3,9 & 542,7 & 12,0 & 463,5 \\
\hline Produits énergétiques & 1372,1 & 68,4 & 8,1 & 0,2 & $-1364,0$ \\
\hline Biens d'équipement & 37,0 & 1,8 & 1484,3 & 32,8 & 1447,3 \\
\hline Biens de consommation & 409,0 & 20,4 & 2415,1 & 53,4 & 2006,1 \\
\hline Métaux précieux/pierres gemmes & 107,7 & 5,4 & 66,1 & 1,5 & $-41,6$ \\
\hline Objets d'art/antiquités & 0,3 & 0,0 & 2,3 & 0,1 & 2,0 \\
\hline Total & $\mathbf{2 0 0 5 , 3}$ & $\mathbf{1 0 0 , 0}$ & $\mathbf{4 5 1 8 , 6}$ & $\mathbf{1 0 0 , 0}$ & $\mathbf{2 5 1 3 , 3}$ \\
\hline
\end{tabular}




\subsection{Composition des échanges de la Suisse avec le tiers-monde (suite)}

D. Principaux exportateurs d'articles manufacturés (2002)

\begin{tabular}{lrrrrr}
\hline \multirow{2}{*}{$\begin{array}{l}\text { Catégorie de } \\
\text { marchandises }\end{array}$} & \multicolumn{2}{c}{ Importations } & \multicolumn{2}{c}{ Exportations } & Solde \\
\cline { 2 - 6 } & Mio Fr. & \% & Mio Fr. & $\%$ & Mio Fr. \\
\hline Matières premières + demi-produits & 2056,0 & 28,7 & 3395,6 & 21,0 & 1339,6 \\
\hline Produits énergétiques & 0,5 & 0,0 & 46,5 & 0,3 & 46,0 \\
\hline Biens d'équipement & 1507,4 & 21,1 & 5280,8 & 32,7 & 3773,4 \\
\hline Biens de consommation & 3139,3 & 43,9 & 5790,3 & 35,9 & 2651,0 \\
\hline Métaux précieux/p. gemmes & 425,4 & 5,9 & 1592,8 & 9,9 & 1167,4 \\
\hline Objets d'art/antiquités & 26,7 & 0,4 & 26,8 & 0,2 & 0,11 \\
\hline Total & $\mathbf{7 1 5 5 , 2}$ & $\mathbf{1 0 0 , 0}$ & $\mathbf{1 6 1 3 2 , 8}$ & $\mathbf{1 0 0 , 0}$ & $\mathbf{8 9 7 7 , 6}$ \\
\hline
\end{tabular}

\section{E. Autres pays du tiers-monde (2002)}

\begin{tabular}{lrrrrr}
\hline \multirow{2}{*}{$\begin{array}{l}\text { Catégorie de } \\
\text { marchandises }\end{array}$} & \multicolumn{2}{c}{ Importations } & \multicolumn{2}{c}{ Exportations } & Solde \\
\cline { 2 - 6 } & Mio Fr. & $\%$ & Mio Fr. & $\%$ & Mio Fr. \\
\hline Matières premières + demi-produits & 466,0 & 17,7 & 864,9 & 17,4 & 398,9 \\
\hline Produits énergétiques & 48,2 & 1,8 & 22,7 & 0,5 & $-25,5$ \\
\hline Biens d'équipement & 107,2 & 4,1 & 1390,9 & 28,0 & 1283,72 \\
\hline Biens de consommation & 1713,4 & 65,1 & 2546,4 & 51,3 & 833,1 \\
\hline Métaux précieux/p. gemmes & 209,2 & 8,0 & 78,9 & 1,6 & $-130,3$ \\
\hline Objets d'art/antiquités & 86,9 & 3,3 & 56,4 & 1,1 & $-30,5$ \\
\hline Total & $\mathbf{2 6 3 0 , 8}$ & $\mathbf{1 0 0 , 0}$ & $\mathbf{4 9 6 0 , 2}$ & $\mathbf{1 0 0 , 0}$ & $\mathbf{2 3 2 9 , 3}$ \\
\hline
\end{tabular}

\section{F. Pays les moins avancés - PMA $^{1}$ (2002)}

\begin{tabular}{lrrrrr}
\hline \multirow{2}{*}{$\begin{array}{l}\text { Catégorie de } \\
\text { marchandises }\end{array}$} & \multicolumn{2}{c}{ Importations } & Exportations & Solde \\
\cline { 2 - 6 } & Mio Fr. & $\%$ & Mio Fr. & \% & Mio Fr. \\
\hline Matières premières + demi-produits & 50,5 & 33,8 & 52,8 & 10,0 & 2,3 \\
\hline Produits énergétiques & 0,0 & 0,0 & 1,6 & 0,3 & 1,6 \\
\hline Biens d'équipement & 0,6 & 0,4 & 293,5 & 55,4 & 292,9 \\
\hline Biens de consommation & 89,9 & 60,1 & 181,5 & 34,2 & 91,6 \\
\hline Métaux précieux/p. gemmes & 6,2 & 4,1 & 0,5 & 0,1 & $-5,7$ \\
\hline Objets d'art/antiquités & 2,3 & 1,5 & 0,2 & 0,0 & $-2,1$ \\
\hline Total & $\mathbf{1 4 9 , 5}$ & $\mathbf{1 0 0 , 0}$ & $\mathbf{5 3 0 , 1}$ & $\mathbf{1 0 0 , 0}$ & $\mathbf{3 8 0 , 5}$ \\
\hline
\end{tabular}

Source: Direction générale des douanes, Statistique du commerce extérieur de la Suisse, Berne, volume I/2002, pp. 854-859.

${ }^{1}$ Pour la liste des PMA, voir la classification du CAD à la fin du présent Annuaire. 
Note: La classification des catégories de marchandises retenue ici a été établie par la Direction générale des douanes. Voici quelques indications concernant sa composition:

- Matières premières et demi-produits: fournitures pour l'agriculture. pour les industries alimentaires et manufacturières, ainsi que pour la construction. Les fournitures les plus importantes sont les demiproduits destinés à l'usage industriel: textiles, ouvrages en bois, en cuir, en caoutchouc ou en plastique, papier. substances chimiques. produits en métal, composants électriques ou électroniques, fournitures d'horlogerie, etc. Une grande partie des matières premières importées en Suisse ou exportées par la Suisse le sont sous la forme de demi-produits. Cette appellation ne signifie pas qu'il s'agit de produits à faible valeur ajoutée. Au contraire, leur fabrication requiert souvent beaucoup de capital, des techniques de pointe et du travail qualifié.

- Produits énergétiques: principalement importations de pétrole brut et de ses dérivés (huiles de chauffage, essence, lubrifiants, etc.). Le charbon et le gaz naturel sont aussi compris dans cette catégorie.

๑ Biens d'équipement: machines et appareils électriques (génératrices, transformateurs, appareils de commande, de signalisation, de contrôle et de mesure, etc.). «non électriques» (moteurs, pompes, compresseurs, machines-outils, machines textiles, etc.), instruments optiques, appareils de mécanique de précision et véhicules utilitaires.

- Biens de consommation: denrées alimentaires, vêtements et chaussures, produits pharmaceutiques, livres, films, meubles, articles et appareils ménagers, radios, téléviseurs, voitures de tourisme, montres, bijoux, jouets, armes et munitions, etc.

- Objets d'art et antiquités: tableaux, peintures et dessins faits à la main, collages, tableautins, gravures, estampes et lithographies, productions originales de l'art statuaire ou de la sculpture, timbres poste, collections d'objets historiques et de sciences naturelles, objets d'antiquités de plus de 100 ans d'âge. 


\subsection{Origine et destination des marchandises échangées entre la Suisse et le tiers-monde, par catégories}

A. Matières premières et demi-produits ${ }^{1}$

1. Importations, exportations et soldes des échanges (2002)

\begin{tabular}{lrrrrrr}
\hline \multirow{2}{*}{ Groupes de pays } & \multicolumn{2}{c}{ Importations } & Exportations & Solde & $\begin{array}{r}\text { Coeff. } \\
\text { échange }\end{array}$ \\
\cline { 2 - 8 } & Mio Fr. & \% & Mio Fr. & \% & Mio Fr. & Exp./Imp. \\
\hline Ensemble du monde & $\mathbf{3 2 2 4 1 , 7}$ & $\mathbf{1 0 0 , 0}$ & $\mathbf{3 2 7 4 2 , 0}$ & $\mathbf{1 0 0 , 0}$ & $\mathbf{5 0 0 , 3}$ & $\mathbf{1 , 0 2}$ \\
\hline Ensemble du tiers-monde & 2601,2 & 8,1 & 4803,1 & 14,7 & 2202,0 & $1,8 \mathbf{5}$ \\
\hline a) Groupes géographiques & & & & & & \\
\hline Afrique & 168,2 & 0,5 & 275,0 & 0,8 & 106,8 & 1,63 \\
\hline Amérique & 578,4 & 1,8 & 842,7 & 2,6 & 264,3 & 1,46 \\
\hline Asie & 1787,3 & 5,5 & 3515,6 & 10,7 & 1728,3 & 1,97 \\
\hline Océanie & 0,4 & 0,0 & 1,4 & 0,0 & 1,0 & 3,50 \\
\hline Europe & 66,8 & 0,2 & 168,4 & 0,5 & 101,6 & 2,52 \\
\hline b) Groupes économiques & & & & & & \\
\hline Export. de pétrole & 79,2 & 0,2 & 542,7 & 1,7 & 463,5 & 6,85 \\
\hline Export. d'art. manuf. & 2056,0 & 6,4 & 3395,6 & 10,4 & 1339,6 & 1,65 \\
\hline Autres pays & 466,0 & 1,4 & 864,8 & 2,6 & 398,9 & 1,86 \\
\hline
\end{tabular}

\section{Principaux fournisseurs et clients (2002)}

\begin{tabular}{|c|c|c|c|c|c|c|c|}
\hline \multicolumn{4}{|c|}{ Principaux fournisseurs } & \multicolumn{4}{|c|}{ Principaux clients } \\
\hline \multicolumn{4}{|c|}{ Importations } & \multicolumn{4}{|c|}{ Exportations } \\
\hline Pays & Mio Fr. & $\%^{2}$ & $\sum \%$ & Pays & Mio Fr. & $\%^{2}$ & $\sum \%$ \\
\hline 1. Chine & 525,8 & 1,63 & 1,63 & 1. Hongkong & 651,3 & 1,99 & 1,63 \\
\hline 2. Brésil & 334,6 & 1,04 & 2,67 & 2. Chine & 546,0 & 1,67 & 3,30 \\
\hline 3. Hongkong & 254,1 & 0,79 & 3,46 & 3. Thailande & 333,7 & 1,02 & 4,32 \\
\hline 4. Thaillande & 243,4 & 0,75 & 4,21 & 4. Turquie & 313,7 & 0,96 & 5,27 \\
\hline 5. Inde & 200,7 & 0,62 & 4,83 & 5. Singapour & 295,6 & 0,90 & 6,18 \\
\hline 6. Taiwan & 117,8 & 0,37 & 5,20 & 6. Brésil & 273,3 & 0,83 & 7,01 \\
\hline 7. Corée du Sud & 92,9 & 0,29 & 5,49 & 7. Taiwan & 251,5 & 0,77 & 7,78 \\
\hline 8. Mexique & 72,3 & 0,22 & 5,71 & 8. Mexique & 224,1 & 0,68 & 8,47 \\
\hline 9. Singapour & 69,2 & 0,21 & 5,93 & 9. Corée du Sud & 218,8 & 0,67 & 9,13 \\
\hline 10. Turquie & 66,9 & 0,21 & 6,13 & 10. Inde & 183,1 & 0,56 & 9,69 \\
\hline 11. Malaisie & 44,3 & 0,14 & 6,27 & 11. Arabie Saoudite & 116,3 & 0,36 & 10,05 \\
\hline 12. Maroc & 40,3 & 0,13 & 6,40 & 12. Emirats Arabes & 94,4 & 0,29 & 10,34 \\
\hline 13. Indonésie & 39,2 & 0,12 & 6,52 & 13. Indonésie & 89,9 & 0,27 & 10,61 \\
\hline 14. Slovénie & 35,9 & 0,11 & 6,63 & 14. Egypte & 74,0 & 0,23 & 10,84 \\
\hline 15. Philippines & 33,9 & 0,11 & 6,73 & 15. Iran & 70,7 & 0,22 & 11,05 \\
\hline Autres pays & 429,8 & 1,33 & 8,07 & Autres pays & 1066,7 & 3,26 & 14,31 \\
\hline Total & 2601,2 & & 8,07 & Total & 4803,2 & & 14,31 \\
\hline
\end{tabular}

${ }^{1}$ La catégorie «Matières premières et demi-produits » est décrite en-dessous des tableaux 1.2., p. 253.

2 Par rapport aux importations et aux exportations totales de matières premières et de demi-produits. 


\subsection{Origine et destination des marchandises échangées entre la Suisse et le tiers-monde, par catégories (suite)}

B. Produits énergétiques ${ }^{1}$

1. Importations, exportations et soldes des échanges (2002)

\begin{tabular}{lrrrrrr}
\hline \multirow{2}{*}{ Groupes de pays } & \multicolumn{2}{c}{ Importations $^{2}$} & Exportations & Solde & $\begin{array}{r}\text { Coeff. } \\
\text { échange }\end{array}$ \\
\cline { 2 - 8 } & Mio Fr. & \% & Mio Fr. & \% & Mio Fr. & Exp./Imp. \\
\hline Ensemble du monde & $\mathbf{5 3 6 8 , 7}$ & $\mathbf{1 0 0 , 0}$ & $\mathbf{3 6 2 , 9}$ & $\mathbf{1 0 0 , 0}$ & $\mathbf{- 5 0 0 5 , 8}$ & $\mathbf{0 , 0 7}$ \\
\hline Ensemble du tiers-monde & 1420,8 & 26,5 & 77,3 & 21,3 & $-1343,4$ & 0,05 \\
\hline a) Groupes géographiques & & & & & & \\
\hline Afrique & 1264,6 & 23,6 & 8,7 & 2,4 & $-1255,9$ & 0,01 \\
\hline Amérique & 0,0 & 0,0 & 2,5 & 0,7 & 2,5 & - \\
\hline Asie & 154,8 & 2,9 & 60,2 & 16,6 & $-94,6$ & 0,39 \\
\hline Océanie & 0,0 & 0,0 & 0,0 & 0,0 & 0,0 & - \\
\hline Europe & 1,4 & 0,0 & 5,8 & 1,6 & 4,4 & 4,14 \\
\hline b) Groupes économiques & & & & & & \\
\hline Export. de pétrole & 1372,1 & 25,6 & 8,1 & 2,2 & $-1364,0$ & 0,01 \\
\hline Export. d'art. manuf. & 0,5 & 0,0 & 46,5 & 12,8 & 46,1 & 101,17 \\
\hline Autres pays & 48,2 & 0,9 & 22,7 & 6,3 & $-25,5$ & 0,47 \\
\hline
\end{tabular}

2. Principaux pays fournisseurs de produits énergétiques, en millions de francs, 2002

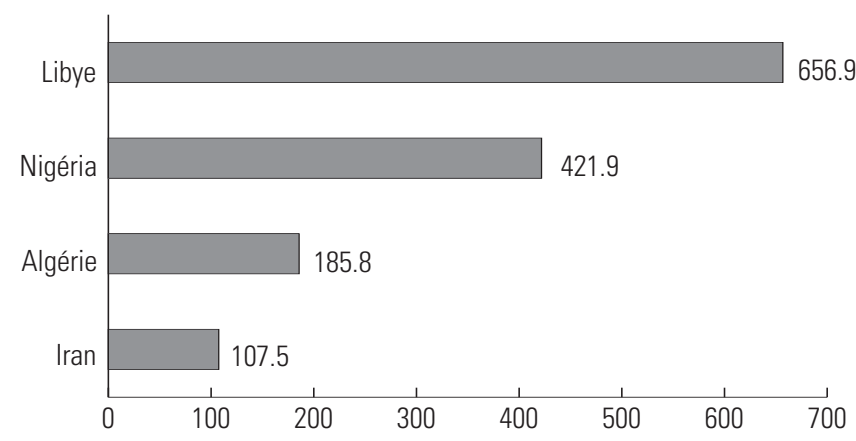

La catégorie «produits énergétiques » est décrite en-dessous des tableaux 1.2., p. 253.

2 Les importations en provenance du tiers-monde concernent essentiellement du pétrole brut acheté aux pays membres de l'OPEP. Le pétrole raffiné chez nos voisins apparaît dans la statistique douanière comme une livraison de ceux-ci (surtout Allemagne, Pays-Bas. France, Belgique et Italie). L'origine effective n'étant pas prise en compte. les importations de produits énergétiques en provenance du tiersmonde semblent donc anormalement faibles (seulement 26,5\% selon les statistiques). 


\subsection{Origine et destination des marchandises échangées entre la Suisse et le tiers-monde, par catégories (suite)}

\section{Biens d'équipement ${ }^{1}$}

1. Importations, exportations et soldes des échanges (2002)

\begin{tabular}{lrrrrrr}
\hline \multirow{2}{*}{ Groupes de pays } & \multicolumn{2}{c}{ Importations } & Exportations & Solde & $\begin{array}{r}\text { Coeff. } \\
\text { échange }\end{array}$ \\
\cline { 2 - 8 } & Mio Fr. & $\mathbf{\%}$ & Mio Fr. & \% & Mio Fr. & Exp./Imp. \\
\hline Ensemble du monde & $\mathbf{3 2 7 0 6 , 0}$ & $\mathbf{1 0 0 , 0}$ & $\mathbf{4 2 2 5 6 , 6}$ & $\mathbf{1 0 0 , 0}$ & $\mathbf{9 5 5 0 , 6}$ & $\mathbf{1 , 2 9}$ \\
\hline Ensemble du tiers-monde & 1651,6 & 5,0 & 8156,0 & 19,3 & 6504,4 & 4,94 \\
\hline a) Groupes géographiques & & & & & & \\
\hline Afrique & 13,1 & 0,0 & 749,2 & 1,8 & 736,1 & 57,19 \\
\hline Amérique & 295,7 & 0,9 & 1190,8 & 2,8 & 895,1 & 4,03 \\
\hline Asie & 1279,4 & 3,9 & 5983,9 & 14,2 & 4704,5 & 4,68 \\
\hline Océanie & 0,2 & 0,0 & 6,1 & 0,0 & 5,9 & 30,50 \\
\hline Europe & 63,1 & 0,2 & 226,0 & 0,5 & 162,9 & 3,58 \\
\hline b) Groupes économiques & & & & & & \\
\hline Export. de pétrole & 37,0 & 0,1 & 1484,3 & 3,5 & 1447,3 & 40,16 \\
\hline Export. d'art. manuf. & 1507,4 & 4,6 & 5280,8 & 12,5 & 3773,4 & 3,50 \\
\hline Autres pays & 107,2 & 0,3 & 1390,9 & 3,3 & 1283,7 & 12,97 \\
\hline
\end{tabular}

\section{Principaux fournisseurs et clients (2002)}

\begin{tabular}{|c|c|c|c|c|c|c|c|}
\hline \multicolumn{4}{|c|}{ Principaux fournisseurs } & \multicolumn{4}{|c|}{ Principaux clients } \\
\hline \multicolumn{4}{|c|}{ Importations } & \multicolumn{4}{|c|}{ Exportations } \\
\hline Pays & Mio Fr. & $\%^{2}$ & $\sum \%$ & Pays & Mio Fr. & $\%^{2}$ & $\sum \%$ \\
\hline 1. Taiwan & 368,7 & 1,13 & 1,13 & 1. Chine & 1245,8 & 2,95 & 2,95 \\
\hline 2. Chine & 339,4 & 1,04 & 2,17 & 2. Turquie & 607,3 & 1,44 & 4,39 \\
\hline 3. Brésil & 262,5 & 0,80 & 2,97 & 3. Corée du Sud & 489,2 & 1,16 & 5,54 \\
\hline 4. Corée du Sud & 152,4 & 0,47 & 3,44 & 4. Taiwan & 468,4 & 1,11 & 6,65 \\
\hline 5. Singapour & 96,5 & 0,30 & 3,73 & 5. Singapour & 451,8 & 1,07 & 7,72 \\
\hline 6. Malaisie & 69,4 & 0,21 & 3,94 & 6. Brésil & 399,6 & 0,95 & 8,67 \\
\hline 7. Hongkong & 56,2 & 0,17 & 4,12 & 7. Hongkong & 348,5 & 0,82 & 9,49 \\
\hline 8. Thailande & 50,7 & 0,15 & 4,27 & 8. Inde & 328,3 & 0,78 & 10,27 \\
\hline 9. Slovénie & 45,2 & 0,14 & 4,41 & 9. Mexique & 324,3 & 0,77 & 11,04 \\
\hline 10. Inde & 37,7 & 0,12 & 4,52 & 10. Malaisie & 273,1 & 0,65 & 11,68 \\
\hline 11. Philippines & 35,9 & 0,11 & 4,63 & 11. Thaillande & 260,0 & 0,62 & 12,30 \\
\hline 12. Turquie & 30,0 & 0,09 & 4,73 & 12. Arabie Saoudite & 239,0 & 0,57 & 12,86 \\
\hline 13. Emirats Arabes & 15,9 & 0,05 & 4,77 & 13. Iran & 220,8 & 0,52 & 13,39 \\
\hline 14. Caïmans & 14,7 & 0,04 & 4,82 & 14. Ethiopie & 155,8 & 0,37 & 13,76 \\
\hline 15. Croatie & 14,2 & 0,04 & 4,86 & 15. Indonésie & 155,3 & 0,37 & 14,12 \\
\hline Autres pays & 62,4 & 0,19 & 5,05 & Autres pays & 2188,8 & 5,18 & 19,30 \\
\hline Total & 1651,6 & & 5,05 & Total & 8156,0 & & 19,30 \\
\hline
\end{tabular}

${ }^{1}$ La catégorie «biens d'équipement» est décrite en-dessous des tableaux 1.2., p. 253.

${ }^{2}$ Par rapport aux importations et aux exportations totales de biens d'équipement. 


\subsection{Origine et destination des marchandises échangées entre la Suisse et le tiers-monde, par catégories (suite)}

D. Biens de consommation ${ }^{1}$

1. Importations, exportations et soldes des échanges (2002)

\begin{tabular}{lrrrrrr}
\hline \multirow{2}{*}{ Groupes de pays } & \multicolumn{2}{c}{ Importations } & Exportations & Solde & $\begin{array}{r}\text { Coeff. } \\
\text { échange }\end{array}$ \\
\cline { 2 - 8 } & Mio Fr. & \% & Mio Fr. & \% & Mio Fr. & Exp./Imp. \\
\hline Ensemble du monde & $\mathbf{5 2 8 0 8 , 6}$ & $\mathbf{1 0 0 , 0}$ & $\mathbf{5 5 0 1 9 , 0}$ & $\mathbf{1 0 0 , 0}$ & $\mathbf{2 2 1 0 , 4}$ & $\mathbf{1 , 0 4}$ \\
\hline Ensemble du tiers-monde & 5261,7 & 10,0 & 10751,8 & 19,5 & 5490,2 & 2,04 \\
\hline a) Groupes géographiques & & & & & & \\
\hline Afrique & 121,3 & 0,2 & 686,0 & 1,2 & 564,7 & 5,66 \\
\hline Amérique & 1320,7 & 2,5 & 2064,2 & 3,8 & 743,5 & 1,56 \\
\hline Asie & 3689,1 & 7,0 & 7654,7 & 13,9 & 3965,6 & 2,07 \\
\hline Océanie & 0,5 & 0,0 & 18,8 & 0,0 & 18,3 & 37,60 \\
\hline Europe & 130,1 & 0,2 & 328,1 & 0,6 & 198,0 & 2,52 \\
\hline b) Groupes économiques & & & & & & \\
\hline Export. de pétrole & 409,0 & 0,8 & 2415,1 & 4,4 & 2006,1 & 5,90 \\
\hline Export. d'art. manuf. & 3139,3 & 5,9 & 5790,3 & 10,5 & 2651,0 & 1,84 \\
\hline Autres pays & 1713,4 & 3,2 & 2546,4 & 4,6 & 833,1 & 1,49 \\
\hline
\end{tabular}

\section{Principaux fournisseurs et clients (2002)}

Principaux fournisseurs

Importations

\begin{tabular}{lrrr}
\hline Pays & Mio Fr. & $\mathbf{\%}^{2}$ & $\sum \%$ \\
\hline 1. Chine & 1332,1 & 2,52 & 2,52 \\
\hline 2. Porto Rico & 611,2 & 1,16 & 3,68 \\
\hline 3. Turquie & 315,5 & 0,60 & 4,27 \\
\hline 4. Corée du Sud & 280,6 & 0,53 & 4,81 \\
\hline 5. Hongkong & 266,1 & 0,50 & 5,31 \\
\hline 6. Colombie & 263,4 & 0,50 & 5,81 \\
\hline 7. Thaillande & 231,3 & 0,44 & 6,25 \\
\hline 8. Inde & 228,1 & 0,43 & 6,68 \\
\hline 9. Arabie Saoudite & 200,4 & 0,38 & 7,06 \\
\hline 10. Vietnam & 124,6 & 0,24 & 7,29 \\
\hline 11. Indonésie & 122,4 & 0,23 & 7,53 \\
\hline 12. Taiwan & 115,1 & 0,22 & 7,74 \\
\hline 13. Brésil & 106,9 & 0,20 & 7,95 \\
\hline 14. Slovénie & 103,2 & 0,20 & 8,14 \\
\hline 15. Mexique & 101,4 & 0,19 & 8,33 \\
\hline Autres pays & 859,4 & 1,63 & 9,96 \\
\hline Total & $\mathbf{5 2 6 1 , 7}$ & & $\mathbf{9 , 9 6}$ \\
\hline
\end{tabular}

Principaux clients

Exportations

\begin{tabular}{lrrr}
\hline Pays & Mio Fr. & $\boldsymbol{\%}^{2}$ & $\sum \%$ \\
\hline 1. Hongkong & 1996,8 & 3,63 & 3,63 \\
\hline 2. Arabie Saoudite & 796,1 & 1,45 & 5,08 \\
\hline 3. Singapour & 731,9 & 1,33 & 6,41 \\
\hline 4. Emirats Arabes & 664,0 & 1,21 & 7,61 \\
\hline 5. Turquie & 644,9 & 1,17 & 8,79 \\
\hline 6. Taiwan & 458,3 & 0,83 & 9,62 \\
\hline 7. Mexique & 441,4 & 0,80 & 10,42 \\
\hline 8. Brésil & 428,1 & 0,78 & 11,20 \\
\hline 9. Corée du Sud & 353,9 & 0,64 & 11,84 \\
\hline 10. Porto Rico & 343,5 & 0,62 & 12,47 \\
\hline 11. Egypte & 263,8 & 0,48 & 12,95 \\
\hline 12. Chine & 253,2 & 0,46 & 13,41 \\
\hline 13. Panama & 198,7 & 0,36 & 13,77 \\
\hline 14. Iran & 188,2 & 0,34 & 14,11 \\
\hline 15. Koweït & 176,5 & 0,32 & 14,43 \\
\hline Autres pays & 2812,6 & 5,11 & 19,54 \\
\hline Total & $\mathbf{1 0 7 5 1 , 7}$ & & $\mathbf{1 9 , 5 4}$ \\
\hline
\end{tabular}

${ }^{1}$ La catégorie «biens de consommation» est décrite en-dessous des tableaux 1.2., p. 253.

${ }^{2}$ Par rapport aux importations et aux exportations totales de biens de consommation. 


\subsection{Origine et destination des marchandises échangées entre la Suisse et le tiers-monde, par catégories (suite)}

E. Métaux précieux et pierres gemmes

1. Importations, exportations et soldes des échanges (2002)

\begin{tabular}{lrrrrrr}
\hline \multirow{2}{*}{ Groupes de pays } & \multicolumn{2}{c}{ Importations } & Exportations & Solde & $\begin{array}{r}\text { Coeff. } \\
\text { échange }\end{array}$ \\
\cline { 2 - 8 } & Mio Fr. & \% & Mio Fr. & \% & Mio Fr. & Exp./Imp. \\
\hline Ensemble du monde & $\mathbf{5 9 6 1 , 8}$ & $\mathbf{1 0 0 , 0}$ & $\mathbf{4 8 0 5 , 9}$ & $\mathbf{1 0 0 , 0}$ & $-\mathbf{1 1 5 5 , 9}$ & $\mathbf{0 , 8 1}$ \\
\hline Ensemble du tiers-monde & $\mathbf{7 4 2 , 3}$ & 12,5 & 1737,8 & 36,2 & 995,5 & 2,34 \\
\hline a) Groupes géographiques & & & & & & \\
\hline Afrique & 13,1 & 0,2 & 10,6 & 0,2 & $-2,5$ & 0,81 \\
\hline Amérique & 137,2 & 2,3 & 22,1 & 0,5 & $-115,1$ & 0,16 \\
\hline Asie & 588,8 & 9,9 & 1684,7 & 35,1 & 1095,9 & 2,86 \\
\hline Océanie & 0,7 & 0,0 & 1,1 & 0,0 & 0,4 & 1,57 \\
\hline Europe & 2,4 & 0,0 & 19,4 & 0,4 & 17,0 & 8,08 \\
\hline b) Groupes économiques & & & & & & \\
\hline Export. de pétrole & 107,7 & 1,8 & 66,1 & 1,4 & $-41,6$ & 0,61 \\
\hline Export. d'art. manuf. & 425,4 & 7,1 & 1592,8 & 33,1 & 1167,4 & 3,74 \\
\hline Autres pays & 209,2 & 3,5 & 78,9 & 1,6 & $-130,3$ & 0,38 \\
\hline
\end{tabular}

\section{Principaux fournisseurs et clients (2002)}

\begin{tabular}{|c|c|c|c|c|c|c|c|}
\hline \multicolumn{4}{|c|}{ Principaux fournisseurs } & \multicolumn{4}{|c|}{ Principaux clients } \\
\hline \multicolumn{4}{|c|}{ Importations } & \multicolumn{4}{|c|}{ Exportations } \\
\hline Pays & Mio Fr. & $\%^{1}$ & $\sum \%$ & Pays & Mio Fr. & $\%^{1}$ & $\sum \%$ \\
\hline 1. Hongkong & 236,9 & 3,97 & 3,97 & 1. Hongkong & 1477,8 & 30,75 & 30,75 \\
\hline 2. Vierges brit. & 94,3 & 1,58 & 5,55 & 2. Thailande & 45,7 & 0,95 & 31,70 \\
\hline 3. Emirats Arabes & 85,2 & 1,43 & 6,98 & 3. Emirats Arabes & 36,9 & 0,77 & 32,47 \\
\hline 4. Thailande & 75,6 & 1,27 & 8,25 & 4. Corée du Sud & 23,2 & 0,48 & 32,95 \\
\hline 5. Liban & 56,4 & 0,95 & 9,19 & 5. Arabie Saoudite & 22,8 & 0,47 & 33,42 \\
\hline 6. Inde & 48,9 & 0,82 & $\overline{10,01}$ & 6. Malte & 19,1 & 0,40 & 33,82 \\
\hline 7. Singapour & 41,5 & 0,70 & 10,71 & 7. Turquie & 15,4 & 0,32 & 34,14 \\
\hline 8. Panama & 22,2 & 0,37 & 11,08 & 8. Vierges brit. & 15,3 & 0,32 & 34,46 \\
\hline 9. Colombie & 12,3 & 0,21 & 11,29 & 9. Liban & 13,1 & 0,27 & 34,73 \\
\hline 10. Arabie Saoudite & 11,8 & 0,20 & 11,49 & 10. Singapour & 9,9 & 0,21 & 34,94 \\
\hline 11. Philippines & 9,5 & 0,16 & 11,65 & 11. Inde & 9,9 & 0,21 & 35,14 \\
\hline 12. Chine & 5,3 & 0,09 & 11,74 & 12. Sri Lanka & 9,7 & 0,20 & 35,35 \\
\hline 13. Kazakhstan & 3,9 & 0,07 & 11,80 & 13. Maurice & 9,4 & 0,20 & 35,54 \\
\hline 14. Algérie & 3,6 & 0,06 & 11,86 & 14. Taiwan & 6,3 & 0,13 & 35,67 \\
\hline 15. Bahamas & 3,3 & 0,06 & 11,92 & 15. Bahamas & 3,6 & 0,07 & 35,75 \\
\hline Autres pays & 31,6 & 0,53 & 12,45 & Autres pays & 19,8 & 0,41 & 36,16 \\
\hline Total & 742,3 & & 12,45 & Total & 1737,8 & & 36,16 \\
\hline
\end{tabular}

${ }^{1}$ Par rapport aux importations et aux exportations totales de métaux précieux et pierres gemmes. 


\subsection{Origine et destination des marchandises échangées entre la Suisse et le tiers-monde, par catégories (fin)}

\section{F. Objets d'art et antiquités}

1. Importations, exportations et soldes des échanges (2002)

\begin{tabular}{lrrrrrr}
\hline & \multicolumn{2}{c}{ Importations } & Exportations & Solde & $\begin{array}{r}\text { Coeff. } \\
\text { échange }\end{array}$ \\
\cline { 2 - 8 } & Mio Fr. & \% & Mio Fr. & \% & Mio Fr. & Exp./Imp. \\
\hline Ensemble du monde & $\mathbf{1 1 0 6 , 5}$ & $\mathbf{1 0 0 , 0}$ & $\mathbf{1 3 3 6 , 6}$ & $\mathbf{1 0 0 , 0}$ & $\mathbf{2 3 0 , 1}$ & $\mathbf{1 , 2 1}$ \\
\hline Ensemble du tiers-monde & 113,9 & 10,3 & 85,5 & 6,4 & $-28,4$ & 0,75 \\
\hline a) Groupes géographiques & & & & & & \\
\hline Afrique & 4,0 & 0,4 & 0,4 & 0,0 & $-3,6$ & 0,10 \\
\hline Amérique & 80,3 & 7,3 & 55,2 & 4,1 & $-25,1$ & 0,69 \\
\hline Asie & 29,5 & 2,7 & 29,6 & 2,2 & 0,2 & 1,01 \\
\hline Océanie & 0,1 & 0,0 & 0,0 & 0,0 & $-0,1$ & 0,00 \\
\hline Europe & 0,1 & 0,0 & 0,3 & 0,0 & 0,2 & 3,00 \\
\hline b) Groupes économiques & & & & & & \\
\hline Export. de pétrole & 0,3 & 0,0 & 2,3 & 0,2 & 2,0 & 7,67 \\
\hline Export. d'art. manuf. & 26,7 & 2,4 & 26,8 & 2,0 & 0,1 & 1,00 \\
\hline Autres pays & 86,9 & 7,9 & 56,4 & 4,2 & $-30,5$ & 0,65 \\
\hline
\end{tabular}

\section{Principaux fournisseurs et clients (2002)}

Principaux fournisseurs

Importations

\begin{tabular}{lrrr}
\hline Pays & Mio Fr. & $\boldsymbol{\%}^{2}$ & $\mathbf{\Sigma} \%$ \\
\hline 1. Bahamas & 64,0 & 5,78 & 5,78 \\
\hline 2. Costa-Rica & 13,1 & 1,19 & 6,97 \\
\hline 3. Hongkong & 11,0 & 1,00 & 7,97 \\
\hline 4. Corée du Sud & 8,3 & 0,75 & 8,72 \\
\hline 5. Chine & 4,0 & 0,36 & 9,08 \\
\hline 6. Liban & 1,4 & 0,13 & 9,21 \\
\hline 7. Egypte & 1,2 & 0,11 & 9,32 \\
\hline 8. Chypre & 1,0 & 0,09 & 9,41 \\
\hline 9. Vierges brit. & 0,9 & 0,08 & 9,49 \\
\hline 10. Rép. dem. Congo & 0,8 & 0,07 & 9,56 \\
\hline 11. Turquie & 0,8 & 0,07 & 9,63 \\
\hline 12. Cap Vert & 0,7 & 0,06 & 9,70 \\
\hline 13. Inde & 0,7 & 0,06 & 9,76 \\
\hline 14. Taiwan & 0,7 & 0,06 & 9,82 \\
\hline 15. Maroc & 0,6 & 0,06 & 9,87 \\
\hline Autres pays & 4,7 & 0,43 & 10,30 \\
\hline Total & $\mathbf{1 1 4 , 0}$ & & $\mathbf{1 0 , 3 0}$ \\
\hline
\end{tabular}

Principaux clients

Exportations

\begin{tabular}{lrrr}
\hline Pays & Mio Fr. & $\boldsymbol{\%}^{2}$ & $\Sigma \%$ \\
\hline 1. Bahamas & 29,8 & 2,23 & 2,23 \\
\hline 2. Costa-Rica & 12,7 & 0,95 & 3,18 \\
\hline 3. Singapour & 10,7 & 0,80 & 3,98 \\
\hline 4. Corée du Sud & 8,6 & 0,64 & 4,62 \\
\hline 5. Vierges brit. & 5,3 & 0,40 & 5,02 \\
\hline 6. Hongkong & 5,1 & 0,38 & 5,40 \\
\hline 7. Porto Rico & 2,6 & 0,19 & 5,60 \\
\hline 8. Panama & 2,0 & 0,15 & 5,74 \\
\hline 9. Brésil & 1,2 & 0,09 & 5,84 \\
\hline 10. Liban & 1,2 & 0,09 & 5,93 \\
\hline 11. Qatar & 1,2 & 0,09 & 6,02 \\
\hline 12. Chypre & 1,0 & 0,08 & 6,10 \\
\hline 13. Colombie & 0,5 & 0,04 & 6,13 \\
\hline 14. Thaillande & 0,5 & 0,03 & 6,17 \\
\hline 15. Venezuela & 0,5 & 0,03 & 6,20 \\
\hline Autres pays & 2,6 & 0,19 & 6,40 \\
\hline Total & $\mathbf{8 5 , 5}$ & & $\mathbf{6 , 4 0}$ \\
\hline
\end{tabular}

${ }^{1}$ La catégorie «objets d'art et antiquités » est décrite en-dessous des tableaux 1.2., p. 253.

${ }^{2}$ Par rapport aux importations et aux exportations totales d'objets d'art et d'antiquités. 